OPEN

SUBJECT AREAS:

MATERIALS SCIENCE

NANOSCIENCE AND

TECHNOLOGY

Received

27 August 2013

Accepted

17 October 2013

Published

5 November 2013

Correspondence and requests for materials should be addressed to

H.Z.W. (wanghz@ dhu.edu.cn) or Y.G.L. lyaogang_li@dhu.edu.

\section{A strong and stretchable self-healing film with self-activated pressure sensitivity for potential artificial skin applications}

\author{
Chengyi Hou', Tao Huang' ', Hongzhi Wang ', Hao Yu', Qinghong Zhang' \& Yaogang Li ${ }^{2}$
}

'State Key Laboratory for Modification of Chemical Fibers and Polymer Materials, College of Materials Science and Engineering, Donghua University, 201620 (People's Republic of China), ${ }^{2}$ Engineering Research Center of Advanced Glasses Manufacturing Technology, College of Materials Science and Engineering, Donghua University, 201620 (People's Republic of China).

Artificial skin, which mimics the functions of natural skin, will be very important in the future for robots used by humans in daily life. However, combining skin's pressure sensitivity and mechanical self-healing properties in a man-made material remains a challenging task. Here, we show that graphene and polymers can be integrated into a thin film which mimics both the mechanical self-healing and pressure sensitivity behavior of natural skin without any external power supply. Its ultimate strain and tensile strength are even two and ten times larger than the corresponding values of human skin, respectively. It also demonstrates highly stable sensitivity to a very light touch $(0.02 \mathrm{kPa})$, even in bending or stretching states.

T he skin is the largest organ in the human body. It is mechanically self-healing and can provide a variable degree of touch sensitivity. It is widely accepted that artificial skin, which mimics the functions of natural skin, will be very important in the future for robots used by humans in daily life for numerous applications. Thus, the development of a thin film material that is flexible and stretchable $e^{1-4}$, sensitive enough to perceive touch $^{1,2,5-8}$, and yet able to heal itself following damage ${ }^{9,10}$ is in high demand in robotic applications. However, combining these properties remains a challenging task: (1) significant progress was made in previous works toward the development of an electronic skin, or pressure sensor, using a variety of materials and physical concepts based on semiconductor technology, but semiconductors lack the capability of emulating the sensitivity and mechanical self-healing ability of natural skin ${ }^{1-3,5-8}$, and (2) artificial self-healing skins have been prepared based on various polymers, but those soft materials are not pressure-sensitive or mechanically strong ${ }^{4,9-12}$. Very recently, Bao and colleagues reported an electrically and mechanically self-healing composite material with pressure-sensitive properties ${ }^{13}$. However, the material demonstrated very low tensile strength $(1 \mathrm{MPa}$, one order of magnitude lower than that of natural human $\operatorname{skin}^{14}$ ) and required an external power supply to demonstrate pressure sensitivity, which is very different to the behavior of real human skin and will limit its potential use in many applications.

Here, we present the first self-healing, mechanically strong and stretchable, self-activated pressure-sensing (abbreviated hereinafter as 5S) device nanostructure. The structure consists of piezoelectric and electrically conductive layers with a healing substrate (see Fig. 1a). The most important component of our material design is the microstructuring of the strong and stretchable electrically self-healing electrode films. In this work, we used a poly ( $N, N$-dimethylacrylamide)-poly(vinyl alcohol)/reduced graphene oxide (PDMAA-PVA/rGO) hybrid as the electrode film. Graphene sheets, because of their mechanical properties and high electrical conductivity, have recently drawn great interest as new nanoscale building blocks to be used to create unique macroscopic materials, such as mechanically strong and highly conductive graphene films ${ }^{15-18}$. However, the ultimate strain of graphene films is only about $0.9 \%$, indicating poor tensile behavior ${ }^{15}$. Liao and coworkers prepared polymer/graphene composite films through the self-assembly of PVA-coated graphene oxide (GO) sheets and subsequent reduction with hydrogen iodide $(\mathrm{HI})^{19}$. The ultimate strain of the sheets produced is $2.67 \%$, but this value is still much lower than that of natural human skin (varies from about 35 to $115 \%$ with the mean value declines linearly from $75 \%$ at birth to $60 \%$ at 90 years with ${ }^{20}$. Very recently, integration of graphene sheets into three-dimensional (3D) macroscopic structures (e.g., porous scaffolds) has attracted considerable attention as an essential step in exploration of the advanced properties of individual $2 \mathrm{D}$ graphene sheets for practical applications $s^{1721-25}$. In our case, preparing flexible and porous graphene foams with interconnected 3D networks is a practical way to help us obtain strong and stretchable electrically self-healing thin films, for the following two reasons: (1) the porous 


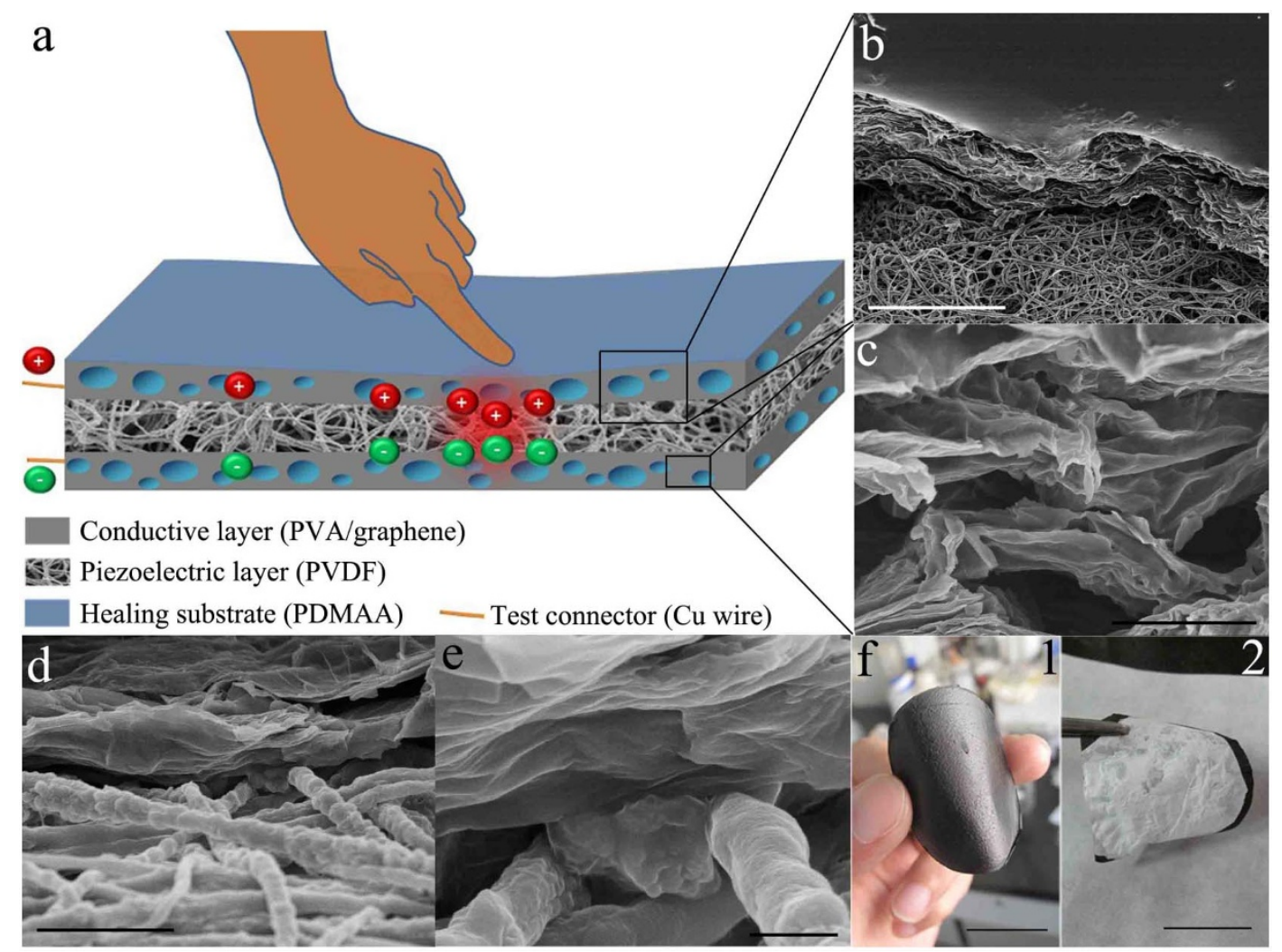

Figure $1 \mid$ The morphologies of the hybrid film. (a) Schematic of the $5 \mathrm{~S}$ film and its pressure sensitivity. Pressures are induced upon the film, and this system detects the total force on the film. (b-e) Cross-sectional FESEM images of the 5S film, showing microscopic structures of the boundary between the two functional layers (b), (d) and (e), and the electrode layer alone (c). Scale bars: $30 \mu \mathrm{m}, 5 \mu \mathrm{m}, 3 \mu \mathrm{m}$ and $500 \mathrm{~nm}$, respectively. (f) Photographs of (1) the hybrid film and (2) the peeled sample. Scale bars: $2 \mathrm{~cm}$.

structure of graphene foams provides higher ultimate strain than graphene films (ref. 26 and Supplementary Fig. S1), and (2) both strengthening and self-healing of 3D graphene materials can be achieved by cross-linking PDMAA chains on the graphene networks, according to our own previous work ${ }^{23}$. Therefore, a 3D PVA/rGO film with interpenetrating PDMAA was used as a strong and stretchable electrically self-healing electrode film in this work. Finally, we prepared self-healing pressure-sensitive hybrid films with electrospun piezoelectric poly(vinylidene fluoride) (PVDF) nanofibers sandwiched between PDMAA-PVA/rGOs. As we know, $\beta$-phase PVDF generates an electrical charge when pressure is applied ${ }^{27-29}$. Combined with outstanding mechanical properties and self-healing abilities of the hybrid films, the pressure sensitivity makes these thin film materials promising candidates for artificial skin applications.

\section{Results}

Fig. 1b-e show cross-sectional field emission scanning electron microscope (FESEM) images of the $5 \mathrm{~S}$ film. As shown in Fig. $1 \mathrm{~b}$ and $c$, the interpenetration of the PDMAA chains leads to a much smoother surface on the inner and outer walls of the 3D graphene networks, when compared to the PVA/rGO porous films (see Supplementary Fig. S2). Interestingly, after treatment with PDM$\mathrm{AA}$, the electrical conductivity of the conductive layer is not remarkably reduced (the mean measured value decreases from 12.5 to $11.2 \mathrm{~S}$ $\mathrm{m}^{-1}$ ), which supports the nanostructure's ability to transmit electrical signals for the sensor application in this work. We also found that the combination of the conductive and piezoelectric layers is strong. As shown in Fig. If and in Supplementary Fig. S3 (control sample), they stand together as a flexible hybrid film and can hardly be peeled off each other without destroying the individual layers. This is mainly attributed to the solid connection between the PVA/ rGO porous film and the PVDF nanofibers. Fig. 1d and e clearly show that PVA/rGO-PVDF junctions were formed at the boundary surfaces of the two functional layers (more junctions are shown in Supplementary Fig. S4). According to previous studies, PVA is miscible with PVDF, and a high degree of intermolecular interaction occurs between the PVDF and PVA chains ${ }^{30,31}$. Also, we observed that without PVA, the PVDF nanofibers would not attach to a pure rGO film. We therefore suggest that the selection of the two polymers is crucial to the structure as a whole.

The surface chemistry of the $5 \mathrm{~S}$ films was studied through their Fourier transform infrared (FTIR) and Raman spectra. Detailed results are shown in the Supplementary Information (see Supplementary Figs. S5 and S6). Both the reduction of GO and the adsorption of the polymer chains are confirmed by the FTIR and Raman results, according to our previously reported work ${ }^{23}$.

More interestingly, the 5S films demonstrate electrically and mechanically self-healing behavior. Fig. 2a depicts a typical electrical healing process. A flake of the film was cut into two pieces, but the electrical conductivity of the flake returned to almost its initial value as soon as the two pieces were brought back into contact (see Fig. 2al and Supplementary Fig. S7). A battery-powered light emitting diode lighting circuit was also constructed to further demonstrate the potential of electrical self-healing for electronic circuits. It should be noted that at this stage, the opposing halves of the film samples were not self-supporting. However, after the film samples had been placed at room temperature in air for $12 \mathrm{~h}$, the film became self-supporting and its ultimate strain and tensile strength recovered almost completely (Fig. 2b1-4). The mean experimentally determined ultimate strain of the $5 \mathrm{~S}$ film over repeated measurements of each sample is $207 \%$, which is the best result ever reported for graphene-based films. Also, the film's mean tensile strength is $91 \mathrm{MPa}$. These values of ultimate strain and tensile strength are approximately two and ten times larger than those of natural human skin, respectively ${ }^{14}$. 

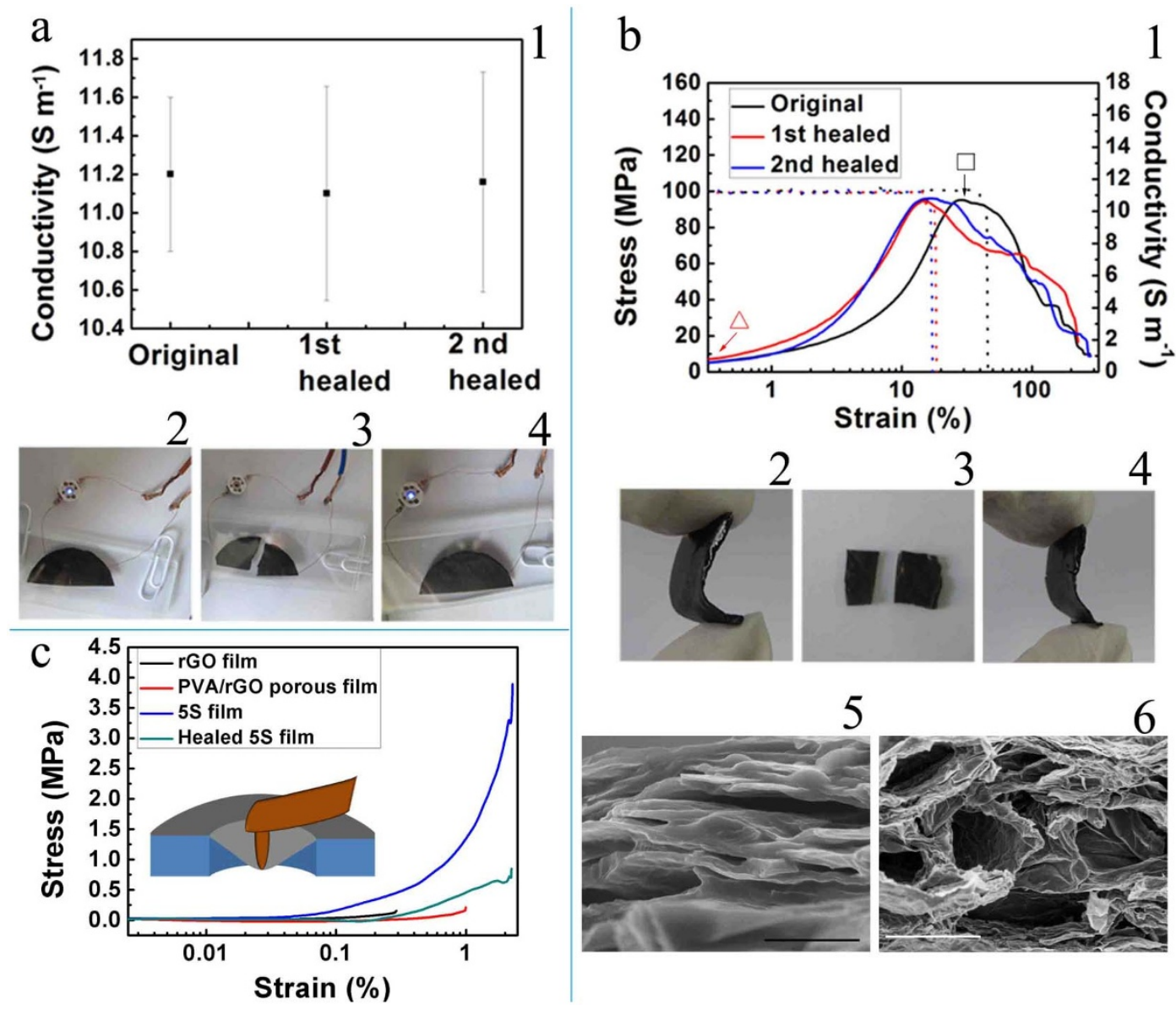

Figure $2 \mid$ Electrical and mechanical self-healing behaviors of the hybrid film. (a) Electrically self-healing behavior characteristics of the 5S film. Error bars are the s.d. calculated from repeated measurements. (b) Mechanically self-healing behavior and corresponding conductivity changes of the $5 \mathrm{~S}$ film, where the full and dotted lines correspond to the stress-strain and conductivity curves, respectively; we used multi-layered 5S films to clearly demonstrate the healing process in photographs (2)-(4); the FESEM images in (5) and (6) correspond to the $\square$ and $\Delta$ states in (1), respectively. Scale bars: $2 \mu \mathrm{m}$ and $5 \mu \mathrm{m}$, respectively. (c) Indentation loading curves of different samples; the inset shows a schematic of the indentation on the suspended film samples.

However, we found that the conductivity of the $5 \mathrm{~S}$ film immediately decreased to zero when it reached the ultimate tensile strength (Fig. 2b1). This can be explained using the FESEM results shown in Fig. $2 \mathrm{~b} 5$ and $2 \mathrm{~b} 6$. During the tensile process, as the film is being stretched, the film's pores tend to close and form a strong graphite-like microstructure (Fig. 2b5). When the conductive PVA/rGO compact layers fracture further, and the $5 \mathrm{~S}$ film starts to lose its conductivity, the whole structure is then only connected by the PDMAA molecules, and thus the stress gradually decreases. Haraguchi and coworkers reported that nanoclay-crosslinked PDMAA hydrogels demonstrate self-healing behavior ${ }^{32}$. In our work, we found that the PDMAA polymer without the structural water and crosslinkers is also self-healing, is even stronger and can recover its shape after deformation (see Supplementary Fig. S8 and Video 1). According to Haraguchi's theory, the macroscopic self-healing of our nanohybrid thin films mainly arises from reconstruction of the network at the interface due to mutual diffusion of PDMAA chains (Supplementary Fig. S9) and their subsequent hydrogen-bonding-driven interactions with each other ${ }^{32}$. Because of the unique properties of the PDMAA component, we observed that the stretched $5 \mathrm{~S}$ film could shrink back to its initial length, and the closed pores in the film are found to open again to recover its $3 \mathrm{D}$ porous structure (Fig. 2b6). This reversible stretching process of 5S film is close to the behavior of natural skin. Meanwhile, the re-stacking of the fractured PVA/rGO sheets also leads to the recovery of the conductivity (see Supplementary Fig. S10 and the dotted lines in Fig. 2b1).

Fig. $2 c$ presents the indentation stress-strain curves of the thin film samples. The force-displacement data were processed to determine the elastic properties and breaking stress of free-standing films. The films were probed by indenting the center of each film with a polytetrafluoroethylene cantilever with a tip radius of $0.72 \mathrm{~mm}$ (Fig. $2 \mathrm{c}$, inset). It was found that the indentation strain of the graphene-based film was improved remarkably by the interpenetrating polymer chains. The mean experimentally determined indentation strength and strain of the $5 \mathrm{~S}$ film are as high as $3.3 \mathrm{MPa}$ and $2.1 \%$, respectively, which is around 13 and 15 times greater than those of the well-known mechanically strong and compact rGO film. After selfhealing of the indentation damage, the indentation strain of the healed $5 \mathrm{~S}$ film measured at the same spot recovers completely, but the mean indentation strength only recovers to $0.76 \mathrm{MPa}$, which is $23 \%$ of the initial value. This is probably due to less efficient recovery at the damaged area in indentation test compared to that in tensile test. However, the value is still $163 \%$ higher than that of the rGO sample. The results show that the device does not crack under a large pressure. In the potential artificial skin application, human bodies and robots will benefit from such protection.

The pressure sensitivity of the $5 \mathrm{~S}$ films was tested using a Keithley 2400 Source Meter at room temperature. The Source Meter was only used to measure the output voltage of the $5 \mathrm{~S}$ films, rather than act as a 
power source. Fig. 3a-d show a series of output curves as a function of external pressure. The sharp positive and negative peaks in the curves in Fig. 3a-c correspond to the pressure and release processes, respectively. The $5 \mathrm{~S}$ films exhibit high sensitivity in low-pressure regimes $(P=0.02-20 \mathrm{kPa})$. The maximum output voltage increased from 0.05 to $0.42 \mathrm{~V}$ with increasing the applied pressure from 0.02 to $20 \mathrm{kPa}$. The output voltage is high enough for sensor applications $\mathrm{s}^{33,34}$. Under larger pressures (20 to $200 \mathrm{kPa}$ ), the maximum output voltage could further increase to around $2 \mathrm{~V}$ (Supplementary Fig. S11). We also measured the output of the $5 \mathrm{~S}$ film under an applied pressure of $0.02 \mathrm{kPa}$ with a reverse connection to the external circuit. Typically, the value remained roughly the same at $0.05 \mathrm{~V}$, but the polarity was reversed (Supplementary Fig. S12), indicating that the measured signals were generated by the $5 \mathrm{~S}$ film rather than the measurement system ${ }^{35,36}$.

The pressure sensitivity of a $5 \mathrm{~S}$ film that healed from being cut was also studied. The pressure dependence of the output voltage of the original and healed films is shown in Fig. 3d. The two curves are well matched, demonstrating that the pressure sensitivity of the $5 \mathrm{~S}$ films can recover completely after the self-healing process, which is mainly due to that the structure of PVDF layer returns (Supplementary Fig. S10) and, more importantly, the PVDF layer generates an electrical charge despite being discontinued following damage, while the electrode genuinely heals.

Next, we examined the output voltage values at different applied pressures when the films were bent or stretched. Fig. $3 \mathrm{e}$ shows the normalized change in the voltage, $\Delta \mathrm{V} / \mathrm{V}_{0}$, where $\Delta \mathrm{V}=\mathrm{V}_{0}-\mathrm{V}_{\mathrm{B}}$; and $\mathrm{V}_{\mathrm{B}}$ and $\mathrm{V}_{0}$ are the mean voltages for the bent and original (i.e. radius of curvature is infinity) films at different applied pressures, respectively, as a function of the bending radius. Clearly, the output voltage shows minimal change on bending with $\Delta \mathrm{V} / \mathrm{V}_{0} \sim 7 \%$ for a radius of curvature of $2.5 \mathrm{~mm}$. Similarly, under stretching conditions, the output voltage also exhibits minimal change, with $\Delta \mathrm{V} / \mathrm{V}_{0} \sim 8 \%$ for an ultimate strain of $10 \%$, where $\Delta \mathrm{V}=\mathrm{V}_{0}-\mathrm{V}_{\mathrm{S}}$; and $\mathrm{V}_{\mathrm{S}}$ and $\mathrm{V}_{0}$ are the voltages at different applied pressures for the stretched and original (i.e. ultimate strain is zero) states, respectively (Fig. 3f). Furthermore, an increase in strain results a more obviously linear-like increase in $\Delta \mathrm{V} / \mathrm{V}_{0}$, compared to the graph trend with increasing bending radius (see Fig. 3e, f, and Supplementary Fig. S14). It could be mainly due to that the deformations of stretched and bent film are distinguished: the film is compressed along the vertical direction of the tensile stress axial line in stretching, while opposite effects occurred at two sides of the film in bending (when the film is bent, one side is stretched while the other is compressed). As a result of which the total precompression (before pressure) of stretching sample was greater than that of bending one, leading to more obviously $\Delta \mathrm{V} / \mathrm{V}_{0}$ changes under pressure.

\section{Discussion}

We have demonstrated a strong and stretchable self-healing thin film material with self-activated pressure sensitivity. This work differs from that of other groups who focus on sensitivity ${ }^{37}$, mechanical properties $^{38-40}$, or self-healing ${ }^{13}$, but miss other vital functions. The strategy used for the design and fabrication of this hybrid film is the integration of nanoscale building blocks into a macroscopic structure, and is currently at the forefront of nanotechnology applications. In our design, the self-healing and pressure sensitivity properties are attributed to PDMAA and PVDF building blocks, respectively. Thus, the device demonstrates similar behaviors to those of the individual components. For example, its self-healing process occurs in air or water at different temperatures and times (Supplementary Fig. S15), which is similar to the behavior of pure PDMAA and PDMAA hydrogels $\mathrm{s}^{32}$; owing to the response time of piezoelectric PVDF layer, the pressure-response curves of the $5 \mathrm{~S}$ films show hysteresis (Fig. $3 \mathrm{a}-$ c). In the magnified views of single cycle (Supplementary Fig. S16), the response time obtained from the double positive peak is found to be about $0.4 \mathrm{~s}$, which is close to other reported values ${ }^{27,28}$. The double peak shown in the single cycle illustrates the process that objects fall
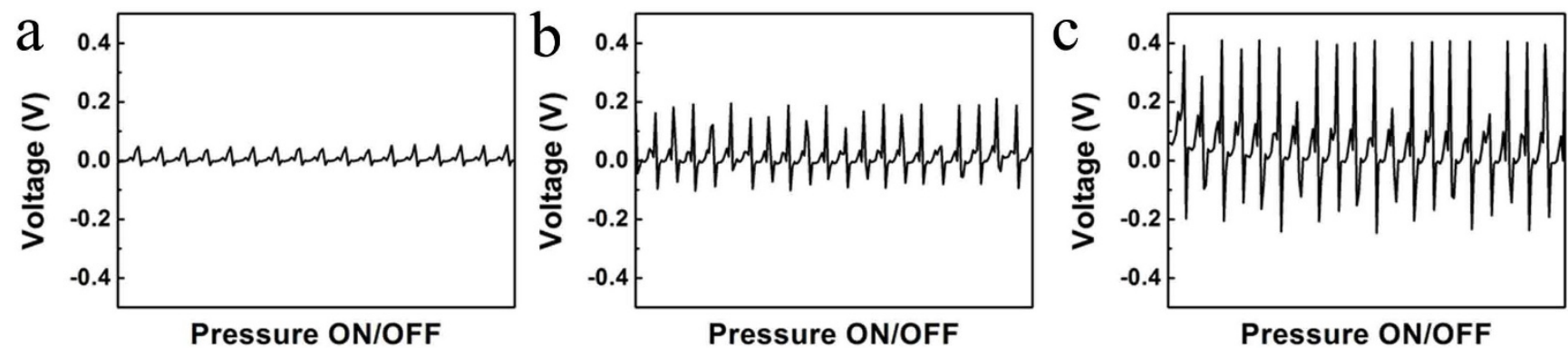

Pressure ON/OFF

Pressure ON/OFF
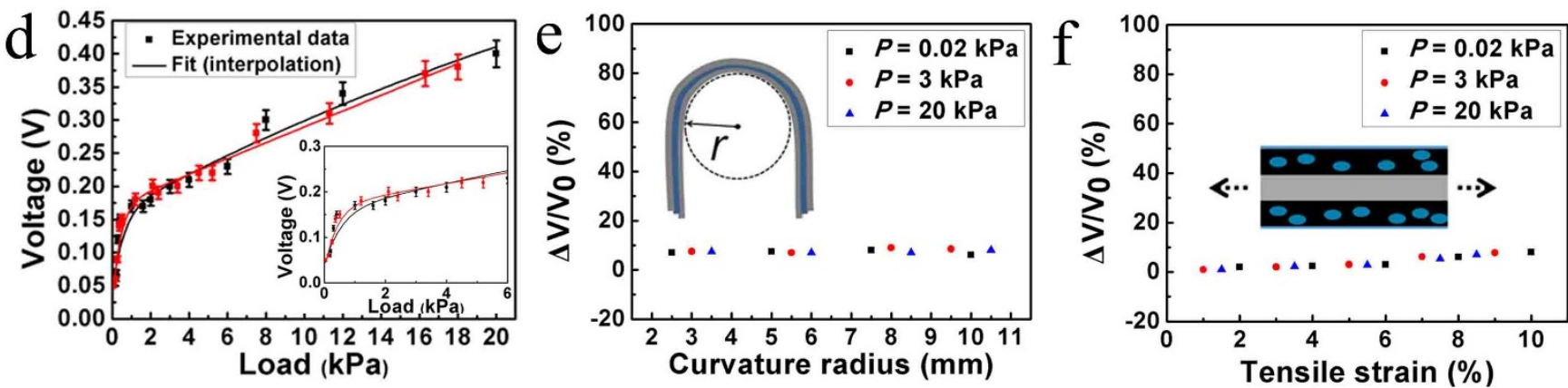

Figure 3 Characterizations of the self-healing electronic sensor skin. $(\mathrm{a}-\mathrm{c})$ Pressure-response curves for the $5 \mathrm{~S}$ film, where the applied pressure $(P)$ was (a) 0.02 , (b) 3 and (c) $20 \mathrm{kPa}$; each experiment lasted $13.3 \mathrm{~s}$, and the output voltage was identified here as the average height of the positive sharp peaks. (d) Output voltage changes under increasing applied pressure (curve fitting report sheets are shown in Supplementary Fig. S13); the black and red data indicate the original sample and the healed sample, respectively. Error bars are the s.d. calculated from repeated measurements. (e), (f) The output voltage change of the $5 \mathrm{~S}$ film at different applied pressures when it has been bent and stretched at different radii of curvature $(r)$ and ultimate strains, respectively; the insets illustrate the bending and stretching orientations in the experiments, pressures were applied at the upper surface of the device illustrated in the insets. 
on the sensor: initially touching the surface of electrodes, and completely acting on the piezoelectric layer when the 3D porous electrode is pressed to form a compact structure (Supplementary Fig. S16). Such detailed observations indicate that our device may have the potential to measure the subtle pressure in real life. In fact, tens of pascals, lower than the typical pressure of a gentle finger touch $(\sim 2 \mathrm{kPa})^{41}$, can be detected by the present device, which compares very favourably with the sensitivity achieved in previous artificial skins: $<0.2 \mathrm{kPa}$ (ref. 1); $<6 \mathrm{kPa}$ (ref. 2); $<\sim 2 \mathrm{kPa}$ (ref. 5); $\sim 1 \mathrm{kPa}$ (ref. 42). Moreover, the device can reliably sense pressure values (Fig. 3a, 3e, $3 \mathrm{f}$ and Supplementary Fig. S17). Its electrical and mechanical reliabilities are comparable to those of previous works ${ }^{1,5,42}$.

However, we notice that the voltage generated by the $5 \mathrm{~S}$ film doesn't show a high degree of stability at a frequency of about $1.5 \mathrm{~Hz}$ (Fig. 3c). It is probably due to the incomplete recovery of 3D structural PDMAA-PVA/rGO electrodes from the compressive deformation during these intervals. As shown in Supplementary Video 1, PDMAA recovers its shape after deformation very gradually. We further investigated the responses of the device under lower cycling frequencies ranging from 0.2 to $1 \mathrm{~Hz}$ for the same applied pressure. With the decrease in the testing frequency, less uneven output signals are recorded (Supplementary Fig. S18). This demonstrates that the device is suitable for use at a range of low frequencies, which is common in real life ${ }^{43}$, for example, in walking, turning over papers and blinking eyes.

The next grand challenge is to combine tactile resolution in our experiment. An interesting path forward is to integrate floating-gate transistor with $5 \mathrm{~S}$ films. By integrating a flexible array of floating-gate transistors with pressure-sensitive rubbers, Takao Someya and his colleagues have fabricated a sensor matrix that detects the spatial distribution of applied pressure ${ }^{44}$. Pressure sensors, source and drain contacts, and capacitors are key components in their device. In order to exploit the self-healing function in such integrated device, one needs to find replacement for each component. The recent achievements in self-healing wires ${ }^{45}$ and self-healing semiconductors ${ }^{46}$ are very encouraging. By employing there materials and our $5 \mathrm{~S}$ films in such design, a self-healing artificial skin that resolves touch profiles is hopefully realized.

In conclusion, we have integrated graphene and polymers into a thin film which mimicked both the mechanical self-healing and pressure sensitivity behavior of natural skin without any external power supply. Its ultimate strain and tensile strength are even two and ten times larger than the corresponding values of human skin, respectively. It also demonstrated high sensitivity to a very light touch, even in bending or stretching states.

\section{Methods}

Synthesis of the 5S film. All reagents were of analytical grade and used as obtained without further purification. GO was synthesized through the Hummers' method $^{47}$. PVDF nanofiber mat (Supplementary Fig. S3) was prepared using an electrospinning technique. In brief, PVDF powders (FR904) were dissolved in a dimethylformamide $(\mathrm{DMF}) /$ acetone mixture $(\mathrm{DMF} /$ acetone $=6 / 4 \mathrm{w} / \mathrm{w})$ at the polymer/solvent weight ratio of 1/9. JG50-1 Model HV power supply (Shanghai Shengfa Detection

Instrument, China) was used in this experiment. The applied voltage was $18 \mathrm{kV}$. The spinning solution was placed in a hypodermic syringe and was delivered to the blunt needle tip at a flow rate of $1 \mathrm{~mL} \mathrm{~h}^{-1}$ via a micro-syringe pump (KDS101, USA) at a fixed collecting distance $(15 \mathrm{~cm})$ between the tip of the syringe and the roller collector. The humidity remained between $30 \%-40 \%$. The PVA/rGO-PVDF-PVA/ rGO sandwiched porous film was prepared by vacuum filtering a PVA (approxi. M.W. 80000)/GO (10 $\left.\mathrm{mg} \mathrm{mL}^{-1}\right)$ solution and another PVA/GO solution containing PVDF nanofiber mat successively, and subsequent reducing the obtained hybrid film in hydrazine vapor $(85 \%)$ at $90^{\circ} \mathrm{C}$ for $12 \mathrm{~h}$. The content of PVA molecules in PVA/ GO film was $20 \mathrm{wt} \%$ so that reduced PVA/GO demonstrated best mechanical properties $^{19}$. The as-prepared hybrid film was immersed into a DMAA solution (containing $9.1 \mathrm{wt} \% 2,2^{\prime}$-Diethoxyacetophenone as initiator) at a relatively low temperature $\left(4^{\circ} \mathrm{C}\right)$ to fill its pores with DMAA monomer. After in situ free-radical polymerization of DMAA, a 5S film was obtained and cut into suitable size. PVA/rGO and PVDF/PVA/rGO porous films and pure PDMAA gel were also prepared via similar processes. An rGO film was prepared as a control sample based on Li's work ${ }^{15}$.
Characterizations and measurements. The morphologies of the as-prepared films were determined at $20 \mathrm{kV}$ using a JSM-6700F FESEM. The electrical conductivity was meausred using a two-probe method. Samples were connected to a Zahner electrochemical workstation (Zennium CIMPS-1) for conductivity measurements. Photographs were taken by a CCD video camera (Canon, PowerShot G10). FTIR spectra were recorded on a Nicolet NEXUS-670 spectrometer. Raman spectra were recorded on a Renishaw in plus laser Raman spectrometer with $\lambda_{\text {exc }}=785 \mathrm{~nm}$. Mechanical measurements were performed using a Dejie DXLL-20000 material testing instrument at room-temperature and a load speed of $2 \mathrm{~mm} \mathrm{~min}^{-1}$. The strain is defined as the change in length, relative to the initial length of the sample. The sample thickness does not impact on the mechanical properties since the unit studied here is $\mathrm{Pa}^{48}$. More detailed discussions of control samples are shown in Supplementary Fig. S19. A SHIMADZU AGS-500ND material testing instrument was used in the indentation test, the films were probed by indenting the center of each film with an accessory polytetrafluoroethylene cantilever with a tip radius of $0.72 \mathrm{~mm}$. Powder X-ray diffraction (XRD) spectroscopy was carried out on a Rigaku $\mathrm{D} / \mathrm{max} 2550 \mathrm{~V} X$-ray diffractometer using $\mathrm{Cu} \mathrm{K} \alpha$ irradiation $(\lambda=1.5406 \AA)$. The operating voltage and current were kept at $40 \mathrm{kV}$ and $300 \mathrm{~mA}$, respectively. SEM images were acquired by a Zeiss Supra VP 40 scanning electron microscope. Optical microscopy and pseudo-color images were obtained using a atomic force microscopy video camera (Agilent Technologies, Chandler, AZ).

In self-healing tests, flakes of the $5 S$ film or pure PDMAA were cut into two pieces, and brought back into contact. Without applied pressure, they were placed at different temperatures from 25 to $80^{\circ} \mathrm{C}$ in air or water for $10 \mathrm{~min}$ to $12 \mathrm{~h}$. The recovery of their electrical and mechanical properties was determined using the methods mentioned above.

Pressure sensitivity of our samples were characterized using a Keithley 2400 Source Meter under room-temperature. Cu wires were used to connect the conductive layers of the $5 \mathrm{~S}$ film and the Source Meter (see Fig. 1a). In experiments, $\mathrm{Cu}$ wires were connected to the upper and lower surfaces (both are conductive layers) of the device. The device was placed flatly on a glass substrate, bent and stretched, respectively. Some tiny objectives such as feather, sponge and quartz glass were utilized to induce pressure upon the device at different frequencies. A Mettler Toledo AL204 laboratory balance was placed under the glass substrate to collect mass data. The pressure was calibrated by free-falling objects at certain heights (see Supplementary Information). All of the testing was carried out in an ambient environment at room temperature.

1. Mannsfeld, S. C. B. et al. Highly sensitive flexible pressure sensors with microstructured rubber dielectric layers. Nature Mater. 9, 859-864 (2010).

2. Someya, T. et al. A large-area, flexible pressure sensor matrix with organic fieldeffect transistors for artificial skin applications. Proc. Natl. Acad. Sci. 101, 9966-9970 (2004).

3. Liu, X., Long, Y.-Z., Liao, L., Duan, X. \& Fan, Z. Large-scale integration of semiconductor nanowires for high-performance flexible electronics. ACS Nano 6 , 1888-1900 (2012).

4. Yannas, I. V. \& Burke, J. F. Design of and artificial skin. I. Basic design principles. J. Biomed. Mater. Res. 14, 65-81 (1980).

5. Takei, K. et al. Nanowire active-matrix circuitry for low-voltage macroscale artificial skin. Nature Mater. 9, 821-826 (2010).

6. Maheshwari, V. \& Saraf, R. F. Tactile devices to sense touch on a par with a human finger. Angew. Chem. Int. Ed. 47, 7808-7826 (2008).

7. Maheshwari, V. \& Saraf, R. F. High-resolution thin-film device to sense texture by touch. Science 312, 1501-1504 (2006).

8. Takahashi, T., Takei, K., Gillies, A. G., Fearing, R. S. \& Javey, A. Carbon nanotube active-matrix backplanes for conformal electronics and sensors. Nano Lett. 11, 5408-5413 (2011).

9. Toohey, K. S., Sottos, N. R., Lewis, J. A., Moore, J. S. \& White, S. R. Self-healing materials with microvascular networks. Nature Mater. 6, 581-585 (2007).

10. Hong, Y. \& Su, M. Multifunctional self-healing and self-reporting polymer composite with integrated conductive microwire networks. ACS Appl. Mater. Inter. 4, 3759-3764 (2012).

11. Lee, K. Y. \& Mooney, D. J. Hydrogels for tissure engineering. Chem. Rev. 101, 1869-1880 (2001).

12. Balazs, A. C. Modeling self-healing materials. Mater. Today 10, 18-23 (2007).

13. Tee, B. C.-K., Wang, C., Allen, R. \& Bao, Z. An electrically and mechanically selfhealing composite with pressure- and flexion-sensitive properties for electronic skin applications. Nature Nanotechnol. 7, 825-832 (2012).

14. Silver, F. H., Siperko, L. M. \& Seehra, G. P. Mechanobiology for force transduction in dermal tissure. Skin Res. Technol. 9, 3-23 (2003).

15. Chen, H., Müller, M. B., Gilmore, K. J., Wallace, G. G. \& Li, D. Mechanically strong, electrically conductive, and biocompatible graphene paper. Adv. Mater. 20, 3557-3561 (2008).

16. Chen, C.-M. et al. Annealing a graphene oxide film to produce a free standing high conductive graphene film. Carbon 50, 659-667 (2012).

17. Yang, X., Zhu, J., Qiu, L. \& Li, D. Bioinspired effective prevention of restacking in multilayered graphene films: towards the next generation of high-performance supercapacitors. Adv. Mater. 23, 2833-2838 (2011).

18. Wang, D.-W. et al. Fabrication of graphene/polyaniline composite paper via in situ anodic electropolymerization for high-performance flexible electrode. ACS Nano 3, 1745-1752 (2009). 
19. Li, Y.-Q., Yu, T., Yang, T.-Y., Zheng, L.-X. \& Liao, K. Bio-inspired nacre-like composite films based on graphene with superior mechanical, electrical, and biocompatible properties. Adv. Mater. 24, 3426-3431 (2012).

20. Edwards, C. \& Marks, R. Evaluation of biomechanical properties of human skin. Clin. Dermatol. 13, 375-380 (1995).

21. Hou, C., Zhang, Q., Li, Y. \& Wang, H. P25-graphene hydrogels: roomtemperature synthesis and application for removal of methylene blue from aqueous solution. J. Hazard. Mater. 205-206, 229-235 (2012).

22. Hou, C., Zhang, Q., Li, Y. \& Wang, H. Graphene-polymer hydrogels with stimulus-sensitive volume changes. Carbon 50, 1959-1965 (2012).

23. Hou, C., Duan, Y., Zhang, Q., Wang, H. \& Li, Y. Bio-applicable and electroactive near-infrared laser-triggered self-healing hydrogels based on graphene networks. J. Mater. Chem. 22, 14991-14996 (2012).

24. Xu, Y., Sheng, K., Li, C. \& Shi, G. Self-assembled graphene hydrogels via a one-step hydrothermal process. ACS Nano 4, 4324-4330 (2010).

25. Tang, Z., Shen, S., Zhuang, J. \& Wang, X. Noble-metal-promoted threedimensional macroassembly of single-layered graphene oxide. Angew. Chem. Int. Ed. 49, 4603-4607 (2010).

26. Niu, Z., Chen, J., Hng, H. H., Ma, J. \& Chen, X. A leavening strategy to prepare reduced graphene oxide foams. Adv. Mater. 24, 4144-4150 (2012).

27. Öğüt, E., Yördem, O. S., Menceloğlu, Y.Z. \& Papila, M. Poly(vinylidene fluoride)/ zinc oxide smart composite material. Proc. of SPIE 6526, Q1-Q10 (2007).

28. Chang, C., Tran, V. H., Wang, J., Fuh, Y.-K. \& Lin, L. Direct-write piezoelectric polymeric nanogenerator with high energy conversion efficiency. Nano Lett. 10, 726-731 (2010).

29. Fang, J., Wang, X. \& Lin, T. Electrical power generator from randomly oriented electrospun poly(vinylidene fluoride) nanofibre membranes. J. Mater. Chem. 21, 11088-11091 (2011).

30. Li, N., Xiao, C., An, S. \& Hu, X. Preparation and properties of PVDF/PVA hollow fiber membranes. Desalination 250, 530-537 (2010).

31. Linares, A., Acosta, J. L., Martinez, A. \& García-Laureiro, J. I. Applications of a statistical model to the analysis of the kinetic parameters in isothermal and nonisothermal crystallization of polymer blends based on PVDF. Polymer 38, 2741-2746 (1997)

32. Haraguchi, K., Uyama, K. \& Tanimoto, H. Self-healing in nanocomposit hydrogels. Macromol. Rapid Comm. 32, 1253-1258 (2011).

33. Yang, Y. et al. Flexible pyroelectric nanogenerators using a composite structure of lead-free $\mathrm{KNbO}_{3}$ nanowires. Adv. Mater. 24, 5357-5362 (2012).

34. Wu, W. et al. Lead zirconate titanate nanowire textile nanogenerator for wearable energy-harvesting and self-powered devices. ACS Nano 6, 6231-6235 (2012).

35. Lin, Z.-H. et al. $\mathrm{BaTiO}_{3}$ nanotubes-based flexible and transparent nanogenerators. J. Phys. Chem. Lett. 3, 3599-3604 (2012).

36. Yang, R., Qin, Y., Dai, L. \& Wang, Z. L. Power generation with laterally packaged piezoelectric fine wires. Nature Nanotechnol. 4, 34-39 (2009).

37. Persano, L. et al. A. High performance piezoelectric devices based on aligned arrays of nanofibers of poly(vinylidenefluoride-co-trifluoroethylene). Nature Commun. 4, 1633 (2013).

38. Park, Y.-L., Chen, B.-R. \& Wood, R. J. Design and fabrication of soft artificial skin using embedded microchannels and liquid conductors. IEEE Sens. J. 12, 2711-2718 (2012).
39. Lacour, S. P., Jones, J., Suo, Z. \& Wagner, S. Design and performance of thin metal film interconnects for skin-like electronic circuits. IEEE Electr. Device L. 25, 179-181 (2004).

40. Cotton, D. P. J., Graz, I. M. \& Lacour, S. P. A multifunctional capacitive sensor for stretchable electronic skins. IEEE Sens. J. 9, 2008-2009 (2009).

41. Dellon, E. S., Mourey, R. \& Dellon, A. L. Human pressure perception values for constant and moving one- and two-point discrimination. Plast. Reconstr. Surg. 90, 112-117 (1992).

42. Ramuz, M., Tee, B. C.-K., Tok, J. B.-H. \& Bao, Z. Transparent, optical, pressuresensitive artificial skin for large-area stretchable electronic. Adv. Mater. 24, 3223-3227 (2012).

43. Xu, S., Hansen, B. J. \& Wang, Z. L. Piezoeletric-nanowire-enabled power source for driving wireless microelectronics. Nature Commun. 1, 93 (2010).

44. Sekitani, T. et al. Organic nonvolatile memory transistors for flexible sensor arrays. Science 326, 1516-1519 (2009).

45. Palleau, E., Reece, S., Desai, S. C., Smith, M. E. \& Dickey, M. D. Self-healing stretchable wires for reconfigurable circuit wiring and 3D microfluidics. Adv. Mater. 25, 1589-1592 (2013).

46. Wang, Y. et al. Self-healing of fractured GaAs nanowires. Nano Lett. 11, 1546-1549 (2011).

47. Hummers, W. S. \& Offeman, R. E. Preparation of graphitic oxide. J. Am. Chem. Soc. 80, 1339 (1958).

48. Chen, C. et al. Self-assembled free-standing graphite oxide membrane. Adv. Mater. 21, 3007-3011 (2009).

\section{Acknowledgments}

The authors thank Yuanlong Shao and Mengye Mao for helpful discussions. This work is supported by NSF of China (No. 51072034, 51172042), MOST of China (2012AA030309), MOE of China (IRT1221, No. 111-2-04), STC of Shanghai (12nm0503900), SRFDP (20110075130001), and Eastern Scholar.

\section{Author contributions}

C.Hou and H.Wang wrote the manuscript. C.Hou, Y.G.Li and H.Wang conceived and designed the experiments. C.Hou and T.Huang performed the experiments. Q.Zhang, H.Yu and Y.Li analyzed the data. All authors reviewed the manuscript.

\section{Additional information}

Supplementary information accompanies this paper at http://www.nature.com/ scientificreports

Competing financial interests: The authors declare no competing financial interests. How to cite this article: Hou, C. et al. A strong and stretchable self-healing film with self-activated pressure sensitivity for potential artificial skin applications. Sci. Rep. 3, 3138; DOI:10.1038/srep03138 (2013).

(c) (i) (2) This work is licensed under a Creative Commons AttributionBY NG SA NonCommercial-ShareAlike 3.0 Unported license. To view a copy of this license, visit http://creativecommons.org/licenses/by-nc-sa/3.0 